REGARDS

SUR L'ECONOMIE ALLEMANDE

BULLETIN ECONOMIQUE DU CRAC

\section{Regards sur l'économie allemande}

Bulletin économique du CIRAC

$82 \mid 2007$

Varia

\title{
Entreprises familiales : un rôle clef outre-Rhin
}

\section{Isabelle Bourgeois}

\section{OpenEdition \\ Journals}

Édition électronique

URL : http://journals.openedition.org/rea/645

DOI : $10.4000 /$ rea. 645

ISBN : 978-2-8218-0860-7

ISSN : 1965-0787

Éditeur

CIRAC

Édition imprimée

Date de publication : 1 juillet 2007

Pagination : 31-34

ISSN : 1156-8992

Référence électronique

Isabelle Bourgeois, «Entreprises familiales : un rôle clef outre-Rhin », Regards sur l'économie allemande [En ligne], 82 | juillet 2007, document 1, mis en ligne le 01 juillet 2009, consulté le 10 décembre 2020. URL : http://journals.openedition.org/rea/645; DOI : https://doi.org/10.4000/rea.645

Ce document a été généré automatiquement le 10 décembre 2020.

(C) CIRAC 


\title{
Entreprises familiales : un rôle clef outre-Rhin
}

\author{
Isabelle Bourgeois
}

1 Non, ce ne sont pas les grands groupes cotés au DAX qui font la compétitivité de l'économie allemande ou tirent la croissance. Au contraire, car ce sont elles qui, en localisant leurs activités à travers le monde entier, créent de l'emploi et de la croissance hors d'Allemagne, comprimant leurs effectifs sur le sol allemand. Entre 2003 et 2005, elles y ont réduit de 3,5\% en moyenne annuelle le nombre d'emplois soumis à cotisations. Si l'emploi non seulement se maintient outre-Rhin, mais accuse aujourd'hui une tendance à la hausse, cela est dû aux entreprises familiales.

\section{$95 \%$ d'entreprises familiales}

2 Cela se comprend aisément : sur les quelque 3,2 millions d'entreprises recensées outreRhin (au CA supérieur à $17500 €$ ), l'écrasante majorité (95,1\%) entre dans la catégorie des familiales. Elles emploient 13,4 millions de salariés $(57,3 \%$ du total des emplois soumis à cotisations sociales) et génèrent un CA de 1900 milliards $€$, soit $41,5 \%$ du total allemand, comme l'explique une étude publiée en avril par l'Institut für Mittelstandsforschung (IfM) de Bonn (Die Volkswirtschaftliche Bedeutung von Familienunternehmen).

\section{Elles sont le cœur du Mittelstand}

3 Ces entreprises familiales constituent ce qu'on appelle le Mittelstand, qui joue un rôle fondamental outre-Rhin dans les activités et, plus encore, le lien social. Elles sont des entités juridiquement indépendantes dont le capital est majoritairement aux mains de la famille fondatrice et qui sont dirigées soit par l'un de ses membres en personne soit par un management extérieur, mais alors sous le contrôle de la famille (la société Stihl dont nous publions le portrait dans ce numéro en est un parfait exemple). Le critère 
appliqué à la définition de cette catégorie est donc qualitatif : il porte sur le mode de gouvernance induit par la structure du capital.

\section{La plupart sont des PME...}

4 Il faut se garder de confondre ces entreprises avec la catégorie des PME, bien que les deux ensembles coïncident largement. Les PME sont en effet définies par deux critères quantitatifs : l'effectif et le CA. Dans la définition allemande (celle de l'IfM), ces seuils sont inférieurs à 500 salariés et à 50 millions $€$; dans la définition de la Commission européenne, ils s'établissent à moins de 250 salariés et à un CA de 50 millions $€$ au maximum (alternative : total bilan limité à 43 millions $€$ ). En vertu de la définition allemande, 99,7 \% des entreprises sont des PME si on considère le CA et même 99,9 \% si on considère l'effectif. Au total, donc, l'Allemagne compte 99,7 \% de PME.

Structure des entreprises allemandes (CA et nombre de salariés) en 2003

\begin{tabular}{|l|c|c|c|c|c|c|}
\hline Chiffre d'affaires & \multicolumn{5}{|c|}{ Nombre de salariés } & Total entreprises \\
\hline & $\mathbf{0 - 9}$ & $\mathbf{1 0 - 4 9}$ & $\mathbf{5 0 - 2 4 9}$ & $\mathbf{2 5 0 - 4 9 9}$ & $>\mathbf{5 0 0}$ & \\
\hline$<1$ million € & 2766697 & 99481 & 13292 & 1555 & 967 & 2881992 \\
\hline de 1 à < 2 millions $€$ & 73899 & 54555 & 1494 & 154 & 134 & 130236 \\
\hline de 2 à < 10 millions $€$ & 37090 & 68866 & 14657 & 401 & 251 & 121265 \\
\hline de 10 à < 25 millions $€$ & 2957 & 6563 & 11802 & 511 & 239 & 22072 \\
\hline de 25 ̀̀ < 50 millions $€$ & 790 & 1181 & 5162 & 1182 & 248 & 8563 \\
\hline$>50$ millions $€$ & 515 & 651 & 2459 & 2182 & 2836 & 8643 \\
\hline Total entreprises & $\mathbf{2 8 8 1 9 4 8}$ & $\mathbf{2 3 1} \mathbf{2 9 7}$ & $\mathbf{4 8 ~ 8 6 6}$ & $\mathbf{5 9 8 5}$ & $\mathbf{4 6 7 5}$ & $\mathbf{3 1 7 2} \mathbf{7 7 1}$ \\
\hline
\end{tabular}

Source : Institut für Mittelstandsforschung, Die volkswirtschaftliche Bedeutung der Familienunternehmen, Bonn, avril 2007 (ifm-Bonn.org). NB : le choix de l'année 2003 s'explique du fait que l'IfM a dû collecter ses données à des sources multiples, et que seules les données de cette année-là sont fiables, car harmonisées.

\section{... mais pas toutes}

5 Cela étant, les entreprises familiales ne sont pas toutes des PME. Elles sont 8643 à dépasser le plafond du CA, et 4675 à occuper plus de 500 salariés. Ce sont elles qui constituent l'objet de l'étude de l'IfM. Partant du constat que les palmarès des 500 plus grosses entreprises allemandes ne distinguent jamais entre les sociétés par actions et celles dont le capital est aux mains de la famille fondatrice, l'institut s'est livré, à la demande la Fondation des entreprises familiales (Stiftung Familienunternehmen; familienunternehmen.de) à une tentative de définition de ces entités méconnues. Et il a publié son propre palmarès, consacré exclusivement aux entreprises familiales. Le 
grand mérite de cette étude est d'apporter des éléments nouveaux permettant de mieux appréhender la structure des entreprises allemandes. En mêlant les traditionnels critères quantitatifs à celui de la propriété du capital et des structures de gouvernance qu'elle induit, elle lève une partie du flou qui entoure le périmètre du Mittelstand, des PME et des entreprises familiales, empêchant désormais l'amalgame volontiers pratiqué entre ces trois catégories.

\section{Les 500 grandes pèsent $11 \%$ du CA total des sociétés allemandes}

6 Les 500 premières entreprises familiales représentaient en 2005 près de $11 \%$ du CA du total des entreprises allemandes et $9 \%$ des emplois soumis à cotisations. Ce sont elles qui dynamisent l'emploi : de 2003 à 2005, elles ont créé 400000 emplois dans le monde, dont la moitié en Allemagne. Elles ont dès lors une fonction éminente "de garants de l'emploi et de la création d'emplois » - tout à l'inverse des entreprises cotées.

Les 10 plus grosses entreprises familiales allemandes

\begin{tabular}{|c|c|c|c|}
\hline Classement par... & $\begin{array}{c}\text { CA (en } \\
\text { millions } €)\end{array}$ & Classement par... & $\begin{array}{l}\text { nombre de } \\
\text { salariés }\end{array}$ \\
\hline 1. Metro AG & 55000 & 1. Metro AG & 257000 \\
\hline 2. BMW AG & 46656 & 2. Robert Bosch & 248853 \\
\hline 3. Robert Bosch & 41461 & 3. BMW AG & 103546 \\
\hline $\begin{array}{l}4 . \quad \text { INA-Schaeffer } \\
\text { Beteiligungsverwaltung GmbH }\end{array}$ & 18989 & 4. Bertelsmann AG & 88516 \\
\hline 5. Bertelsmann AG & 17890 & 5. Fresenius Aktiengesellschaft & 71611 \\
\hline $\begin{array}{l}6 . \quad \text { Henkel } \quad \text { Erste } \\
\text { Verwaltungsgesellschaft } \mathrm{mbH}\end{array}$ & 13060 & $\begin{array}{lcr}6 . & \text { Henkel } \quad \text { Erste } \\
\text { Verwaltungsgesellschaft } \mathrm{mbH}\end{array}$ & 62129 \\
\hline 7. $\mathrm{CH}$ Boehringer Sohn, Ingelheim & 9535 & $\begin{array}{l}7 . \quad \text { INA-Schaeffer } \\
\text { Beteiligungsverwaltung GmbH }\end{array}$ & 60000 \\
\hline 8. HERAUS Holding $\mathrm{GmbH}$ & 9311 & 8. Adolf Würth GmbH \& Co. KG & 50767 \\
\hline 9. Fresenius Aktiengesellschaft & 7889 & 9. $\mathrm{CH}$ Boehringer Sohn, Ingelheim & 37406 \\
\hline 10. Dr. August Oetker KG & 7029 & $\begin{array}{l}\text { 10. Freudenberg Simrit GmbH \& } \\
\text { Co. KG }\end{array}$ & 33385 \\
\hline
\end{tabular}

Source des données : ibid. NB : Ne figurent ici que les données 2005. 


\title{
Certaines sont cotées en bourse, mais toujours sous contrôle familial
}

7 Cette listes des 500 principales familiales comprend également les discounters Aldi et Lidl \& Schwarz, les distributeurs Tengelmann Warenhandelsgesellschaft KG, Karstadt Quelle AG ou Otto, des banques comme la Sal. Oppenheim jr.\& Cie. Kommanditgesellschaft auf Aktien ou encore des prestataires de services de santé comme le groupe Marseille-Kliniken AG. Si certaines sont des sociétés par actions cotées en bourse (Metro AG, BMW AG notamment), leur capital ou la politique du groupe n'en restent pas moins contrôlés par la famille propriétaire, souvent via des fondations, comme cette Quandt-Stiftung à la tête de BMW ou cette Fondation Mohn à la tête de Bertelsmann (société non cotée). Il en va de même des groupes Aldi ou Lidl (voir REA 67/04).

\begin{abstract}
"Les groupes familiaux, un phénomène typiquement allemand" (Handelsblatt)

Le quotidien économique Handelsblatt, l'un de ces supports qui publient régulièrement une série de palmarès, dont dernièrement celui des 50 sociétés non cotées d'Europe ayant connu la plus forte hausse de leur CA au cours de l'exercice écoulé (édition du 22-05-07), s'est intéressé lui aussi de près à la structure de la propriété de ces sociétés.

Il identifie, quant à lui, comme leader allemand le groupe Haniel né voici 250 ans (effectif : 55000 ; CA : 27,7 milliards $€$ ). Le capital de ce conglomérat structuré en cinq divisions (Celesio: distribution pharmaceutique et services; ELG : recycling et matières premières ; HTS : propreté ; TAKKT : B2B ; Xella : matériaux et systèmes de construction) appartient à plus de 500 actionnaires, dont le premier est de loin le groupe Metro (18,6\%). Si, aux dires de la société (www.haniel.de), son code de gouvernance prescrit la séparation des fonctions de propriété et de management, «les échanges entre la famille et la direction sont multiples "; la famille propriétaire pilote en effet le conseil de surveillance, et si elle se réserve $25 \%$ des bénéfices, le reste est systématiquement réinvesti dans le développement des activités.

Le quotidien présente non sans fierté les résultats de ce palmarès où figure en tête le suisse Glencore (matières premières ; CA : 88,5 milliards $€$ ), suivi du néerlandais Vitol (pétrole; 86,5 milliards $€$ ) et du groupe allemand Schwarz (Lidl et autres ; 44 milliards $€$ ), les groupes Auchan et Intermarché se situant respectivement en $8^{\mathrm{e}}$ et $9^{\mathrm{e}}$ position. Il écrit ainsi à propos de ces groupes : non seulement "près de la moitié d'entre eux a son siège en République fédérale ", mais ils ont aussi "une santé resplendissante». Certes, leur part dans le total des entreprises nationales est à peine supérieure à ce qu'elle est en France, en Espagne ou en Italie, il n'empêche que 29 d'entre eux sont allemands : «les groupes familiaux sont un phénomène typiquement allemand " s'intitule l'article. Et il constate que c'est outre-Rhin que se concentrent «pratiquement tous les grands groupes d'Europe qui ne sont spécialisées ni dans la distribution ni dans le commerce des matières premières "».
\end{abstract}

\section{Quatre GmbH (sarl) sur cinq sont des familiales}

8 Le critère qualitatif permettant de définir une entreprise familiale - l'identité entre propriété du capital et management - se décline en effet de plusieurs manières. Aucune statistique officielle n'existant en la matière, l'IfM s'est appuyé sur les diverses bases de 
données d'entreprises existant en Allemagne (dont Hoppenstedt et Markus) pour calculer la part des familiales dans chacune des catégories de statut juridique. Si ces calculs reposent sur des données recueillies entre 1998 et 2000, elles ont non seulement le mérite d'exister, mais leurs enseignements restent d'actualité : la structure du tissu des entreprises allemandes étant stable (comme le révèlent dans un autre contexte les statistiques fiscales), les ordres de grandeur publiés par l'IfM ne devraient pas avoir varié significativement depuis. Il ressort ainsi de cette étude que $28,3 \%$ des sociétés par actions (AG) sont contrôlées directement ou indirectement par la famille. Dans le cas des sociétés à responsabilité limitée $(\mathrm{GmbH})$, cette part s'élève à 79,9\%. Les sarl en commandite ( $\mathrm{GmbH} \& \mathrm{Co} . \mathrm{KG}$ ) sont $85,6 \%$. La part du contrôle familial est supérieure encore dans la catégorie des sociétés en commandite (KG: 96,8\%) et des sociétés en nom collectif ou de droit civil (respectivement OHG et GbR: 95,5\%). Seules les entreprises en nom personnel sont à $100 \%$ familiales, ce qui tombe sous le sens, ce statut étant la version la plus pure de l'identité entre propriété et gouvernance. Si on réunit tous ces statuts, alors la part des entreprises familiales au sens où les définit l'IfM s'élève à $94,5 \%$ du total des entreprises allemandes. Cette approche corrobore les résultats obtenus en fonction d'autres critères ou sur d'autres bases, comme ceux de Destatis. Pour l'office (Wirtschaft und Statistik, 4/2007) qui, rappelons-le, ne prend pas en considération les critères qualitatifs développés par l'IfM, les deux tiers $(63,5 \%)$ des entreprises allemandes recensées en 2004 sont en nom personnel, 16,5\% des sociétés à capitaux ( $\mathrm{GmbH}, \mathrm{AG}$, etc.) et 11,6\% des sociétés de personnes (OHG, KG, etc.), les 8,4\% restants rassemblant les autres statuts (dont les coopératives). Mais les statistiques officielles relatives aux entreprises, trop grossières et donc peu opératoires en matière de politique économique (fiscalité des entreprises, soutien au Mittelstand...) sont en cours de révision, et la réforme devrait entrer en vigueur en 2011.

\section{Les entreprises familiales sont d'abord industrielles}

9 L'étude de l'IfM corrobore d'un point de vue scientifique et structurel les constats établis par la presse économique et plus particulièrement le palmarès du quotidien Handelsblatt. Elle révèle que, sur les 500 grosses entreprises familiales allemandes figurant dans sa liste à lui, 270 appartiennent au secteur de l'industrie. Et elles sont fortement internationalisées. Cette appartenance à l'industrie est une caractéristique foncière des entreprises familiales allemandes en général. On y trouve $83,5 \%$ d'entreprises industrielles; leur part dans l'emploi est de $42 \%$, elles génèrent $30 \% \mathrm{du}$ CA de l'industrie et contribuent pour un tiers aux investissements. Il s'agit pour la plupart de PME dans la définition allemande. Ce groupe comprend bien sûr des entreprises artisanales et s'étend aux services aux entreprises ; il n'est plus possible de les séparer, puisqu'elles oeuvrent dans le même secteur.

Part des entreprises familiales dans l'industrie (2001)

\begin{tabular}{|c|c|c|c|}
\hline Salariés & Nombre d'entreprises industrielles... & $\ldots$ dont familiales & (part) \\
\hline 1 à 19 & 79216 & 70428 & $88,9 \%$ \\
\hline 20 à 49 & 6874 & 5774 & $84,0 \%$ \\
\hline
\end{tabular}




\begin{tabular}{|c|c|c|c|}
\hline 50 à 99 & 7521 & 5626 & $74,8 \%$ \\
\hline 100 à 249 & 7965 & 5104 & $64,1 \%$ \\
\hline 250 à 499 & 2721 & 1311 & $48,2 \%$ \\
\hline 500 à 999 & 1251 & 438 & $35,0 \%$ \\
\hline$>1000$ & 850 & 151 & $17,8 \%$ \\
\hline Total & 106398 & 88832 & $83,5 \%$ \\
\hline
\end{tabular}

Source des données : ibid.

\section{La propriété reste la valeur-clef de l'entrepreneuriat}

10 Autrement dit, les données minutieusement collectées et réunies par l'IfM pour la période 1998-2000 révèlent que «l'idée prédominante selon laquelle serait caractéristique pour le secteur industriel le grand groupe conduit par un management salarié est fausse en ce qui concerne l'industrie allemande. Dans l'industrie aussi, comme dans tous les autres secteurs de l'économie, prédominent en effet les entreprises familiales qui se sont souvent développées dans la continuité de plusieurs générations ».

Entreprises industrielles en Allemagne - données-clefs (2001)

\begin{tabular}{|l|c|c|c|l|}
\hline Entreprises... & $\begin{array}{c}\text {... dirigées par la } \\
\text { famille propriétaire }\end{array}$ & $\begin{array}{c}\text {... par un } \\
\text { management } \\
\text { salarié }\end{array}$ & $\begin{array}{c}\text { Total entr. } \\
\text { indus. }\end{array}$ & Familiales \\
\hline Nombre & 88832 & 17566 & 106398 & $83,5 \%$ \\
\hline Emplois & 3197302 & 4419600 & 7616902 & $42,0 \%$ \\
\hline CA (milliards $€$ ) & 451 & 1075 & 1525 & $29,5 \%$ \\
\hline $\begin{array}{l}\text { Investissements } \\
(\text { milliards } € \text { ) }\end{array}$ & 22 & 49 & 71 & $31,2 \%$ \\
\hline
\end{tabular}

Source des données : ibid. NB : chiffres arrondis par nos soins.

11 Comparées aux entreprises du Dax ou du M-Dax, les 500 grosses familiales se distinguent par plusieurs aspects. Elles occupent plus de salariés: 9,2\% du total allemand, contre $6,7 \%$ seulement. Et elles ont créé de l'emploi $(+1,6 \%$ sur la période $2003 / 04$, puis $+8,1 \%$ sur $2004 / 05$, soit $+4,8 \%$ en moyenne), alors que les sociétés cotés en détruisaient ( $-1,7 \%$ en moyenne entre 2003 et 2005). Sur la même période, l'emploi total reculait de $-1,5 \%$ en moyenne annuelle outre-Rhin. Par ailleurs, leur CA 
enregistrait une hausse supérieure au cours de ces années ( $+7,5 \%$ en moyenne annuelle contre $+4,4 \%)$.

\section{Elle explique la compétitivité du Mittelstand allemand...}

Comment expliquer ces disparités? Assurément, les entreprises familiales ont profité de la croissance de la demande mondiale pour investir de nouveaux marchés et développer de nouveaux produits ou procédés - facteur de croissance traditionnel du Mittelstand industriel allemand, quel que soit le périmètre considéré. Mais la principale raison, selon l'IfM, est à chercher dans "ce lien très fort entre les propriétaires et leur entreprise » qui «se traduit par une grande flexibilité et une forte capacité d'adaptation, $y$ compris dans un environnement économique difficile». Visiblement, ajoute l'institut, ces entreprises savent, pour s'adapter, mettre à profit "une perception proactive des opportunités se présentant à l'échelle mondiale " qu'elles saisissent en développant leurs activités à l'étranger, ce qui se répercute par une hausse de leur CA et de leurs effectifs en Allemagne.

\section{... et sa résistance à une gouvernance centrée sur la seule valorisation boursière}

13 Pour le dire autrement: ce lien étroit nourrit un engagement plus prononcé du propriétaire qui «développe un intérêt profond pour le développement durable de son entreprise ", nettement plus, en tout cas, que ne peut le faire le management salarié d'une société par action, humainement plus anonyme. Quant à la flexibilité, elle n'est que la résultante du mode de gouvernance induit, qui se traduit par des processus décisionnels nettement plus courts. On le voit, le modèle allemand de l'entreprise, bien que largement globalisé au niveau des process, ce dont il tire sa compétitivité, est loin de se montrer perméable à une logique purement financière et centrée sur le court terme. La prédominance des structures familiales s'y oppose largement, contrairement à ce que laisse présumer un discours public qui voit le "capitalisme rhénan " frappé d'obsolescence face à une gouvernance de type shareholder value frappé du sceau valorisant de la globalisation financière.

\section{INDEX}

Mots-clés : croissance, entreprise familiale, Mittelstand, PME 